\title{
Electronic Health Record Goes Personal World-wide
}

\author{
YC Li' ${ }^{1}$, PS Lee ${ }^{2}$, WS $\mathrm{Jian}^{3}$, CH Kuo ${ }^{4}$ \\ 'Graduate Institute of Biomedical Informatics, Taipei Medical University, Taipei, Taiwan \\ ${ }^{2}$ Institute of Biomedical Informatics, National Yang-Ming University, Taipei, Taiwan \\ ${ }^{3}$ School of Health Care Administration, Taipei Medical University, Taipei, Taiwan \\ ${ }^{4}$ Department of Information Management, National Taiwan University, Taipei, Taiwan
}

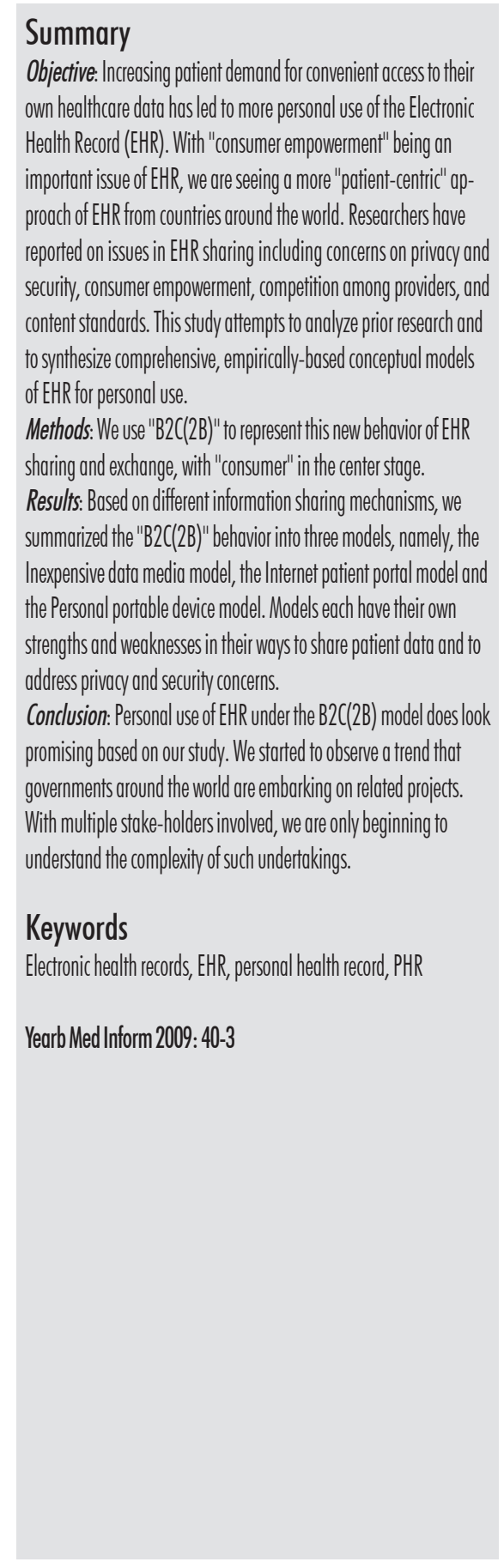

\section{Background}

The evolution from an institutional Computerized Patient Record (CPR) to a longitudinal and cross-institution Electronic Health Record (EHR), and further to a Personal Health Record (PHR) that integrates life-style, nutritional and self-measured health parameters is becoming a pervasive concept in recent years. An EHR system enables physicians to view patients' data from multiple sources across a period of time for comprehensiveness and continuity of care. On the other hand, increasing patient demand for convenient access to their own healthcare data has led to more personal use of the Electronic Health Record (EHR) [1,2]. With "consumer empowerment" being an important issue of EHR, we are seeing a more "patient-centric" approach of EHR from countries around the world. Studies show that proper use of EHR can decrease medical errors $[3,4]$, facilitate the detection of adverse health events [5], enable more appropriate use of healthcare services [6], and potentially lower healthcare costs $[7,8]$. However, EHR adoption has been slow. Researchers have reported on issues related to EHR sharing, including concerns on privacy and security, consumer empowerment, competition among providers, and content standards. This study attempts to analyze prior research and to synthesize comprehensive, empirically-based conceptual models of EHR for personal use. Personal use of EHR is an important first step in creating effective and measurable interventions to personal health maintenance. In e-commerce terms, there are two basic exchange models for EHR information, namely "B2B-Business to Business" and "B2CBusiness to Customers". "B2B" represents the exchange between two healthcare providers for the purpose of continuity of care. "B2C" refers to the sharing of EHR to the patients themselves. From there, patients may review their own EHR, consult with people they trust or take this "portable" EHR to the next provider. We can denote this behavior of taking patients' own EHR to the next provider as "C2B". By connecting "B2C" and "C2B", the whole process can be described as "B2C2B". Since the last part " $2 \mathrm{~B}$ " is optional, we use "B2C (2B)" to represent this new model of EHR sharing and exchange. We can also call it "Patient-Initiated Exchange" (PIX) because the exchange process is mostly evoked and controlled by the patients.

\section{Methods}

We review papers and case-studies for those EHR systems used nation-wide, or those designed for nation-wide use. This study does not include demonstrations or feasibility studies done at a small scale. The literature we reviewed is mainly in English, so it is possible that we may overlook literature published in other languages.

\section{Models for Personal Use of EHR}

There are many different ways to facilitate personal use of EHR. Based on 


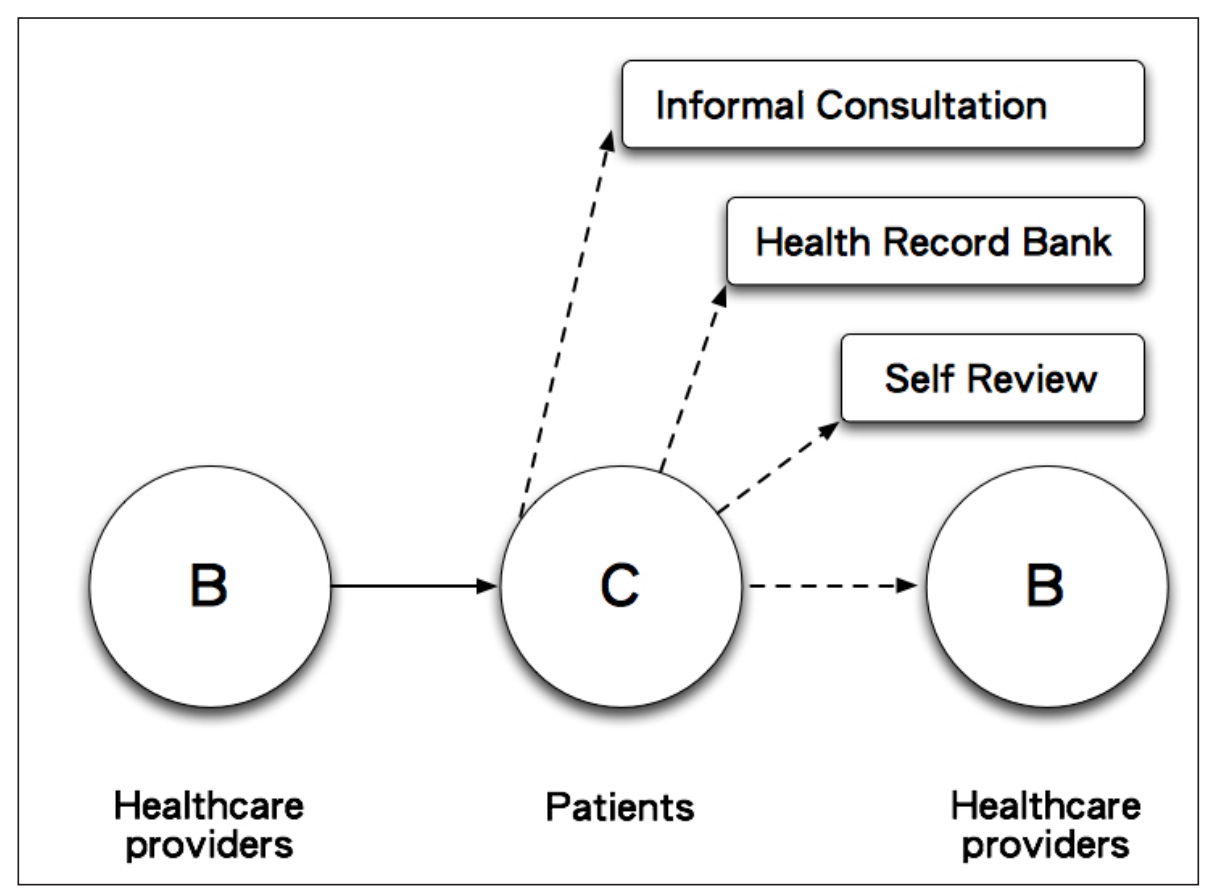

Fig. 1 The Patient-Initiated Exchange (PIX) model for EHR sharing and exchange through patients

different information sharing mechanisms, we summarized them into three models. The Inexpensive data media model, the Internet patient portal model and the Personal portable device model. Each model has its own strengths and weaknesses with different ways of addressing privacy and security concerns (Table 1).

Table 1 A comparison of the three models of EHR sharing and exchange with patients

\begin{tabular}{|c|c|c|c|}
\hline & Inexpensive data media model & Internet patient portal model & Personal portable device model \\
\hline Media & $\begin{array}{l}\text { Flash memory, CD-ROM, Smart } \\
\text { Card, etc. }\end{array}$ & None & $\begin{array}{l}\text { PDA, cell phone, smart phone and } \\
\text { Ultra Mobile PC (UMPC) }\end{array}$ \\
\hline Advantages & $\begin{array}{l}\text { 1. Low media cost } \\
\text { 2. Small in size and highly } \\
\text { portable; } \\
\text { 3. Good privacy }\end{array}$ & $\begin{array}{l}\text { 1. Easy access } \\
\text { 2. No physical media }\end{array}$ & $\begin{array}{l}\text { 1. Better privacy than the Internet } \\
\text { portal model } \\
\text { 2. Easy to update } \\
\text { 3. Personal reminders possible }\end{array}$ \\
\hline Disadvantages & $\begin{array}{l}\text { 1. Can not be viewed without } \\
\text { additional viewer and viewing } \\
\text { device } \\
\text { 2. Not ease to update } \\
\text { 3. Easy to be misplaced }\end{array}$ & $\begin{array}{l}\text { 1. Need Internet connection } \\
\text { 2. Need a reliable and trusted } \\
\text { back-end health information } \\
\text { service provider } \\
\text { 3. Privacy and security issues } \\
\text { abound }\end{array}$ & $\begin{array}{l}\text { 1. Bulkier } \\
\text { 2. More expensive to own and } \\
\text { maintain }\end{array}$ \\
\hline Examples & $\begin{array}{l}\text { MERIT-9 project (Japan); TMT } \\
\text { project (Taiwan); Smart Card } \\
\text { (Taiwan, Germany, Malaysia) }\end{array}$ & $\begin{array}{l}\text { Electronic Patient Folder } \\
\text { (Germany); MyHealthOnLine } \\
\text { (UK); Danish National Health } \\
\text { Portal (Denmark) }\end{array}$ & $\begin{array}{l}\text { TET / TrEHRT (APAMI, AMIA, EFMI, } \\
\text { IMIA) }\end{array}$ \\
\hline
\end{tabular}

mission medium of medical images and medical summaries to patients who apply for them. DICOM images on a CDROM compliant to the DICOMDIR standard are also commonly supported by major PACS (Picture Archiving and Communication Systems) vendors in Japan as well as other countries. (2) Taiwan's TMT (Taiwan Electronic medical record Template) project $[10,11]$ uses USB sticks as the primary media for hospitals to share non-imaging EHR information with their patients. (3) In addition, a small amount of more secure information-sharing can be achieved by Smart Card as a medium. Notable examples can be found in Taiwan, Germany and Malaysia. Taiwan's Health Smart Card carried by its 23 million population store diagnoses, medications, allergy and vaccination information among other insurance-related data. (4) German national health IT body (Gematik) [12] is also issuing a patient Smart Card for core medical data and considering using a USB stick to store the rest of EHR data. (5) Malaysia MyKAD is a government multipurpose Smart Card that also will support some personal medical data [13].

The advantages of this model are: (1) low media cost (2) small in size and highly portable (3) better privacy because they are carried personally. However, information stored on the portable media cannot be viewed without additional equipment. The user will need a computer, a viewer application and, when using Smart Cards, a card reader. The information is not easy to update and, if there are many different viewers for the same type of data, as in the DICOMDIR CD-ROM case, the users can get confused and become reluctant to use them because they are unable to familiarize themselves with so many different viewers. A patient may also misplace the portable media, causing concerns on privacy breach.

\subsection{Internet Patient Portal Model}

An intuitive way to provide personal use of EHR is through an Internet patient portal. Using the Internet to pro- 
vide information storage and user-interface, a patient will be able to access personal health information with a web browser. Electronic Patient Folder in Germany plans to offer a longitudinal, person-related medical history by using their health card as a key to identify, authenticate and authorize access. In the UK, patients will be able to access a summary of their own health and care information, known as the Summary Care Record (SCR), via portal services such as MyHealthOnLine [14]. Denmark uses a "Danish National Health Portal" to provide EHR information access for patients and healthcare professionals [15]. Prominent examples from the industry on patient portal would include Google's "Google Health" and Microsoft's "Health Vault". Although both projects have larger plans in the future, they do provide functional web-based patient portals for depositing health information and for "importing" EHR information from participating healthcare providers.

This model offers fast updating and easy access to the users. Compared to the "Inexpensive data media" model, this model frees the users from carrying a physical medium and therefore eliminates the risk of misplacing it. On the other hand, a user must be able to access the Internet and a reliable/ trusted back-end health information service provider (governments or non-profit organizations are preferred). The individual's privacy and security issues will have to be addressed properly by the health information service providers before this model can be accepted by the general public.

\subsection{Personal Portable Device Model}

Popular personal portable devices such as PDAs, cell phones, smart phones and Ultra Mobile PCs (UMPCs) allow immediate access to personal health information either stored locally in the device, or remotely in a server, (which can be readily downloadable through a live connection). Combined with intelligent applications, this model can pro- vide powerful personal reminders for a patient. Common examples include medication reminders and alerts (such as drug-drug interactions), health checkup and life-style reminders. An international collaborative project - TET / TrEHRT (Traveler's EHR Template) is a practical example of this model $[11,16]$. Based on the open Android operating system from Google, they developed a JAVA-based viewer for a summarized patient record designed for travelers. This viewer, which runs on Google phones, offers two modes of storage for the personal health data: off-line and online. In the off-line mode, the data are stored locally in the memory of the phone and can be exported to an XML file compatible to the CCR (Continuity of Care Record) standard developed by ASTM in the US [17]. In the online mode, the data are stored remotely in a server and can be downloaded through a secure Internet connection. This model provides better privacy than the Internet portal model and is easier to update than the Inexpensive data media model. Unlike the Internet portal model, a personal portable device can operate even without a network connection (when data are stored in the device). However, such devices are bulkier than most flash memory gadgets and more expensive to own and maintain.

\subsection{Security and Privacy}

Security and privacy are among the highest priority issues when health information sharing are considered. During the whole sharing process, we must ensure the integrity and non-repudiation of the shared information. A public key infrastructure (PKI) is generally considered crucial to achieve a safe environment for health information sharing. Some countries use dedicated PKI for health information processing to retain better security and privacy. An increasing number of examples can be found around the world including the Health Certificate Authority (HCA) Card in Taiwan, the Health Card (eGK) in Germany, the UZIcard [18] in the Netherlands.

\section{Discussion}

The patients are open to the use of EHR, but they have concerns about privacy and security [19]. They want their privacy to be respected, and the systems to be safe from the intrusion of outsiders. The model of "B2C(2B)" exchange is potentially a solution for it, because this model shifts control of personal health information back to the patient her/himself and thus resolved the data ownership problem and also alleviated the patients' concern on how the data will be used among healthcare providers. However, important steps must be taken before this model can be fully realized:

(1) An environment that guarantees the integrity and non-repudiation of the health information exchange or sharing is required. As described above, PKI is a popular and feasible solution today. However, the cost of building a nation-wide PKI can be formidable and would require longterm planning and maintenance.

(2) In order to exchange data between providers and to share consistently with the patients, a common content standard has to be available. For example, Taiwan's electronic Medical record Template (TMT) is being implemented in major hospitals in Taiwan as a common content standard. With TMT, healthcare providers are able to provide to their patients a set of standardized digitally-signed XML files that contain a patient's EHR information. More than 2,000 patients have received their own EHR which they can browse with a free viewer. The CCR is also a potential content standard for a summary of patient data.

(3) Support from the healthcare organizations is essential for the B2C(2B) model to be successful. A precise mapping process and a datamapping gateway are required initial investment from the provider side in order to transform the standard patient file from their internal data structure. This would also involve a set of standard vocabularies among 
the providers, in addition to the format of the contents shared.

\section{Conclusion}

Personal use of EHR under the B2C(2B) model does look promising based on our study. We started to observe a trend that governments around the world are embarking on related projects. With multiple stake-holders involved, we are only beginning to understand the complexity of such undertakings.

It can be difficult for the patients to really read and understand their own healthcare data. Managing the data using computers and Internet tools poses another challenge, especially for the elderly, who happen to generate more data about their health and healthcare.

The sharing of EHR with the patients may also trigger cautions for some physicians. There are physicians who believe that "too much" information can fuel malpractice suits. On the other hand, some believe that personal use of EHR may actually alleviate the problem because physicians will be more willing to discuss with the patients when they know that the information will eventually be shared, and that better-informed patients file less complaints [10].

Important factors that influence a provider to adopt the "B2C(2B)" model are quality and competition. On the quality side, this model allows a closer look of the medical record by the patients. It may give patients more confidence on the quality of care or inspire doubt if the information is incomplete and/or inaccurate. Competition among the providers can also determine the speed and scope of adoption. Competing hospitals may want to belay the sharing of the patient records to prevent losing their patients to competitors. On the other hand, they cannot afford not to do it if their competitors have already started to share the EHR with the patients since the inaction can be interpreted as having something to hide.
An array of opportunities will be opened to the industry when most patients carry their own personal EHR. For example, a "health record bank" as proposed by Ball et al. can be a starting point for more "value-added" services provided by the enterprise [20, 21]. The B2C(2B) model can also help clinical research by offering more secondary use of patient data. Hypothetically speaking, the data could be collected through a virtual "Facebook for Clinical Trial" on popular Internet social network. It may attract people with the same health problems to gather and share their personal EHR with the clinical trials that they trust.

Although the possibilities seem endless, it will take some time for all the stake-holders to understand and embrace this new model. It is going to be a long but worthy journey towards prevalent personal use of EHR. With all the new incentives from major countries around the world to facilitate Health Information Technology adoption, we believe that the day that we "liberate" health information back to the hands of the people is near.

\section{Acknowledgement}

This research is partially supported by the National Science Council grant NSC 97-3114-E010-001 and the Department of Health, Executive Yuan, Taiwan.

\section{References}

1. Siteman E, Businger A, Gandhi T, Grant R, Poon E, Schnipper J, et al. Clinicians Recognize Value of Patient Review of their Electronic Health Record Data. AMIA Annu Symp Proc 2006;2006:1101.

2. Halamka JD, Mandl KD, Tang PC. Early experiences with personal health records. J Am Med Inform Assoc 2008 Jan-Feb;15(1):1-7.

3. Bates DW, Cohen M, Leape LL, Overhage JM, Shabot MM, Sheridan T. Reducing the frequency of errors in medicine using information technology. J Am Med Inform Assoc 2001;8:299-308.

4. Overhage JM, Tierney WM, Zhou XA, McDonald CJ. A randomized trial of corollary orders to prevent errors of omission. J Am Med Inform Assoc 1997;4:364-75.

5. Bates DW, Evans RS, Murff H, Stetson PD, Pizziferri L, Hripcsak G. Detecting adverse events using information technology. J Am Med Inform Assoc 2003;10:115-28.
6. Bates DW. Physicians and ambulatory electronic health records. Health Aff. 2005;24:1180-9.

7. Hillestad R, Bigelow J, Bower A, Girosi F, Meili R, Scoville R, et al. Can electronic medical record systems transform health care? Potential health benefits, savings and costs. Health Aff 2005;24:1103-17.

8. Lobach DF, Detmer DE. Research challenges for electronic health records. Am J Prev Med 2007 May;32(5 Suppl):S104-11.

9. EHR Global Perspective August 2008 version 1.0.1. Accessed June 7, 2009, from http://www.himss.org content/files/200808_EHRGlobalPerspective_ whitepaper.pdf

10. Jian WS, Hsu CY, Hao TH, Wen HC, Hsu MH, Lee YL, et al. Building a portable data and information interoperability infrastructure-framework for a standard Taiwan Electronic Medical Record Template. Comput Methods Programs Biomed 2007 Nov;88(2):102-11.

11. Establishing a Standardized Travelers' Electronic Health Summary Template (TET) for the Asia-Pacific Region. Accessed June 7, 2009, from http:// www.medinfo.org.tw/tet/

12. Wendling D. The German eHealth programme. Card Technology Today 2009 January;21(1):10-1.

13. MyKAD-ONLINE: Bridging the gap to your future. Accessed June 7, 2009, from https:// www.mykadonline.com.my/mykad.html.

14. Greenhalgh T, Wood GW, Bratan T, Stramer K, Hinder S. Patients' attitudes to the summary care record and HealthSpace: qualitative study. BMJ 2008 Jun 7;336(7656):290-5. Epub 2008 May 29

15. Lippert S, KvernelandA. The Danish National Health Informatics Strategy. Stud Health Technol Inform 2003;95:845-50.

16. IMIA-APAMI-AMIA-EFMI Project - TrEHRT Viewer Demo. Accessed June 7, 2009, from http:/ /www.youtube.com/watch?v=ROsaFHZaWGs

17. ASTM International: Standards Worldwide. Accessed June 6, 2009, from http://www.astm.org/

18. Care for safe and reliable information. Accessed June 7, 2009, from http://www.uziregister.nl/Images/UZI-folder_per_pagina_engels_tcm 3817238.pdf

19. Zurita L, Nøhr C. Patient opinion-EHR assessment from the users perspective. Stud Health Technol Inform 2004;107(Pt 2):1333-6.

20. Health Record Banking Alliance. Retrieved June 7, 2009, from http://www.hhs.gov/healthit/ahic/materials/06_07/cps/hrba.pdf

21. Gold JD, Ball MJ. The health record banking imperative: a conceptual model. IBM Systems Journal 2007;46(1):43 - 55

Correspondence to:

Yu-Chuan (Jack) Li

Graduate Institute of Biomedical Informatics

Taipei Medical University

250 Wu-Xin Street

Taipei, Taiwan 110

Tel: +886227361661 ext 2069

Fax: +886223787795

E-mail: jack@tmu.edu.tw 